\title{
On the number of Arnoux-Rauzy words
}

\author{
by
}

Filippo Mignosi (Palermo) and Luca Q. Zamboni (Denton, TX)

1. Introduction. Let $\omega=\omega_{1} \omega_{2} \ldots$ be a sequence with values in a finite alphabet $A$. The complexity function $p_{\omega}: \mathbb{N} \rightarrow \mathbb{N}$ assigns to each $n$ the number of distinct factors (or subwords) of $\omega$ of length $n$. A fundamental result due to Hedlund and Morse states that a sequence $\omega$ is ultimately periodic if and only if for some $n$ the complexity $p_{\omega}(n) \leq n$. (See [11], [25] and [22, Chapter 2].) Sequences of complexity $p(n)=n+1$ are called Sturmian sequences or Sturmian words (see [22, Chapter 3]). The best known example is the Fibonacci sequence

$12112121121121211212112112121121121211212112112121121 \ldots$

fixed by the morphism $1 \mapsto 12$ and $2 \mapsto 1$. It is well known that all Sturmian words can be realized geometrically by an irrational rotation on the circle (see $[11,25])$. More precisely, every Sturmian word is obtained by coding the symbolic orbit of a point $x$ on the circle (of circumference one) under a rotation by an irrational angle $\alpha$ where the circle is partitioned into two complementary intervals, one of length $\alpha$ and the other of length $1-\alpha$. And conversely every such coding gives rise to a Sturmian word. The irrational number $\alpha$ is called the slope.

Let $\mathrm{St}_{n}$ denote the cardinality of the set of all Sturmian words $u$ of length $n$, that is, the set of words $u$ of length $n$ in $\{0,1\}$ which are a factor of some Sturmian sequence in $\{0,1\}^{\mathbb{N}}$. In [24], Mignosi gave a proof of an explicit formula for $\mathrm{St}_{n}$ in terms of the Euler phi function $\varphi(n)$, conjectured by Dulucq and Gouyou-Beauchamps [13] (see also Corollary 4). A combinatorial proof of this formula was given by de Luca and Mignosi in [23] (see also [22, Chapter 3]). In this paper we describe a multidimensional generalization of the Euler phi function which counts the number of Arnoux-Rauzy words of each length. 
Definition 1. Let $A_{k}=\{1, \ldots, k\}$ with $k \geq 2$. A sequence $\omega$ in the alphabet $A_{k}$ is called an Arnoux-Rauzy sequence if it satisfies the following three conditions:

- $\omega$ is recurrent,

- the complexity function $p_{\omega}(m)$ equals $(k-1) m+1$,

- for each $m$ there is exactly one right special and one left special factor of $\omega$ of length $m$.

Recall that a factor $u$ of $\omega$ is called right special (resp. left special) if $u$ is a prefix (resp. suffix) of at least two words of length $|u|+1$ which are factors of $\omega$. A word which is both right and left special is called bispecial. Arnoux-Rauzy sequences are a natural generalization of Sturmian words; Sturmian words correspond to taking $k=2$ in the above definition. For $k=3$ the combinatorial conditions listed in Definition 1 distinguish them from other sequences of complexity $2 n+1$ such as those obtained by coding trajectories of 3-interval exchange transformations [16, 17, 18], or those of Chacon type, i.e., topologically isomorphic to the subshift generated by the Chacon sequence [6, 15]. Perhaps the best known example on three letters is the so-called Tribonacci sequence defined as the fixed point of the morphism $\tau(1)=12, \tau(2)=13$ and $\tau(3)=1$. In [26] Rauzy showed that the subshift generated by $\tau$ is isomorphic (in measure) to an exchange of three fractal domains in $\mathbb{R}^{2}$ which generate a tiling of the plane.

Arnoux and Rauzy [2] showed that each Arnoux-Rauzy sequence may be geometrically realized by an exchange of $2 k$ intervals on the circle, and is uniquely ergodic. It was further believed, as in the case of Tribonacci and the Rauzy fractal, that each Arnoux-Rauzy sequence is measure isomorphic to a rotation on the torus, i.e., is obtained by a symbolic coding of the trajectories of points under a rotation on the $k$-dimensional torus with respect to a natural partition. This was recently disproved by Cassaigne-FerencziZamboni in [4] where the authors exhibited an Arnoux-Rauzy sequence $\omega$ on a 3-letter alphabet $\{0,1,2\}$ which is totally unbalanced in the following sense: for each $n>0$ there exist two factors of $\omega$ of equal length, with one having at least $n$ more occurrences of the letter 0 than the other. It follows that the cylinder [0] is not a bounded remainder set (in the sense of Kesten [21]) and hence via an unpublished result of Rauzy later generalized by Ferenczi [14], either $\omega$ is not a natural coding of a rotation in $\mathbb{R}^{n}$ modulo a lattice, or the $\mathrm{A}-\mathrm{R}$ sequence $\omega(0)$, obtained by coding $\omega$ according to first returns to 0 , is not a natural coding of a rotation in $\mathbb{R}^{n}$ modulo a lattice.

Arnoux-Rauzy sequences have been extensively studied from many different points of view in connection with dynamical systems (see $[1,2,7,8$, $20]$ ), number theory (see $[7,9,19,20,27,29,30]$ ) and combinatorics (see [4, $5,7,12,20,27])$. 
Acknowledgements. The second author was supported in part by a grant from the Texas Advanced Research Program, and from the NSF. We wish to thank the referee for useful comments and suggestions.

2. Counting words. We fix $k \geq 2$ and let $\mathcal{A R}_{\infty}=\mathcal{A} \mathcal{R}_{\infty}(k)$ be the set of all Arnoux-Rauzy sequences on the alphabet $A_{k}$. We denote by $\mathcal{A R}$ the set of all Arnoux-Rauzy words, that is, the set of all words $u$ (including the empty word) such that $u$ is a factor of some Arnoux-Rauzy sequence $x \in \mathcal{A R}_{\infty}$. For each $n \geq 0$ we let $\mathcal{A R}_{n}$ be the set of all $u \in \mathcal{A R}$ of length $n$.

For each $a \in A_{k}$ define the morphism $\tau_{a}$ on $A_{k}$ by $\tau_{a}(a)=a$ and $\tau_{a}(b)=$ $a b$ for all $b \in A_{k}$ different from $a$. Then it is proved in [2] (see also [27]) that each Arnoux-Rauzy sequence $\omega$ is in the shift orbit closure of a unique sequence of the form

$$
\omega_{*}=\lim _{j \rightarrow \infty} \tau_{i_{1}} \circ \ldots \circ \tau_{i_{j}}(1)
$$

where the sequence of indices $\left(i_{j}\right)$ (called the coding sequence) takes values in $A_{k}$. Moreover each $a \in A_{k}$ occurs in the coding sequence an infinite number of times. The sequence $\omega_{*}$ is called a characteristic Arnoux-Rauzy sequence.

Lemma 1. Let $u \in \mathcal{A R}$ and suppose that for some $b, c \in A_{k}$ distinct, $u b$ and uc are in $\mathcal{A R}$. Then there exists an Arnoux-Rauzy sequence $\omega \in \mathcal{A R}_{\infty}$ which contains as factors the $k$ words $u 1, u 2, \ldots, u k$. In other words if $u$ is a right special factor of $\mathcal{A R}$ then $u$ is a right special factor of some ArnouxRauzy sequence $\omega$.

Proof. We proceed by induction on the length of $u$. The result is clearly true if $u$ is empty, or if $|u|=1$. Writing $u=a v$ with $a \in A_{k}$ and $|v| \geq 1$, we make the inductive hypothesis that the result of the lemma holds for all words of length smaller than $|u|$. Thus $a v b$ and $a v c$ are each in $\mathcal{A R}$. Without loss of generality we can assume 1 is the last letter of $v$.

CASE 1: $a=1$. If $a v$ is of the form $a v=1^{n}$, then for each ArnouxRauzy sequence $\omega$, the Arnoux-Rauzy sequence $\tau_{1}^{n}(\omega)$ contains the $k$ words $1^{n+1}, 1^{n} 2,1^{n} 3, \ldots, 1^{n} k$. Next suppose that $a v$ is not of the form $1^{n}$. If $b$ and $c$ are each different from 1 , then we can write $a v b=1 v b=\tau_{1}\left(v^{\prime} b\right)$ and $a v c=$ $1 v c=\tau_{1}\left(v^{\prime} c\right)$ for some $v^{\prime}$ with $v^{\prime} b, v^{\prime} c \in \mathcal{A R}$ and $\left|v^{\prime}\right|<|u|$. By the inductive hypothesis there exists an Arnoux-Rauzy sequence $\omega$ which contains both $v^{\prime} b$ and $v^{\prime} c$ as factors. It follows that the Arnoux-Rauzy sequence $\tau_{1}(\omega)$ contains both $a v b$ and $a v c$ as factors. Hence $a v$ is a right special factor of $\tau_{1}(\omega)$.

Next assume that one of $b$ or $c$ (say $b$ ) is equal to 1 . Then $a v b=1 v 1=$ $1 v^{\prime} 11$ and $a v c=1 v c=1 v^{\prime} 1 c$ for some $v^{\prime}$ in $\mathcal{A R}$. Thus we can write $1 v^{\prime} 1=$ $\tau_{1}\left(v^{\prime \prime} 1\right)$ and $1 v^{\prime} 1 c=\tau_{1}\left(v^{\prime \prime} c\right)$ for some $v^{\prime \prime}$ with $v^{\prime \prime} 1, v^{\prime \prime} c \in \mathcal{A R}$ and $\left|v^{\prime \prime}\right|<$ $|u|$. By the inductive hypothesis there exists an Arnoux-Rauzy sequence 
$\omega$ which contains both $v^{\prime \prime} 1$ and $v^{\prime \prime} c$ as factors. Thus $\tau_{1}(\omega)$ contains both $1 v^{\prime} 1=\tau_{1}\left(v^{\prime \prime} 1\right)$ and $1 v^{\prime} 1 c=\tau_{1}\left(v^{\prime \prime} c\right)$. As $\tau_{1}$ of each letter begins with 1 , it follows that $1 v^{\prime} 11$ is also a factor of $\tau_{1}(\omega)$. Hence $1 v^{\prime} 1=a v$ is a right special factor of $\tau_{1}(\omega)$.

CASE 2: $a \neq 1$. In this case it is easy to see that $1 a v b$ and $1 a v c$ are both in $\mathcal{A R}$. Applying the arguments of Case 1 we deduce that $1 a v$ is a right special factor of some Arnoux-Rauzy sequence $\omega$. Hence so is $a v$.

As an immediate consequence of Lemma 1 we have

Corollary 1. Let $r(n)$ denote the number of right special factors of $\mathcal{A R}$ of length $n$. Then

$$
\operatorname{Card}\left(\mathcal{A R}_{n}\right)=\operatorname{Card}\left(\mathcal{A R}_{n-1}\right)+(k-1) r(n-1) .
$$

Proof. In fact each right special factor of length $n-1$ is a prefix of $k$ factors of length $n$.

Lemma 2. Suppose $u \in \mathcal{A R}$ is a bispecial factor of $\mathcal{A R}$, that is, there exist letters $a \neq b$ and $c \neq d$ such that $a u, b u, u c, u d$ are in $\mathcal{A R}$. Then there exists an Arnoux-Rauzy sequence $\omega \in \mathcal{A R}_{\infty}$ such that $u$ is a bispecial factor of $\omega$.

Proof. The proof of Lemma 2 is similar to the proof of Lemma 1: using the $\tau_{i}$ the result follows by induction on the length of the words.

Lemma 3. If $u$ is a bispecial factor of an Arnoux-Rauzy sequence, then for each $a \in A_{k}$ there exists an Arnoux-Rauzy sequence $\omega$ such that $u$ is a bispecial factor of $\omega$ and au is a right special factor of $\omega$.

Proof. Let $\nu=\tau_{n_{1}} \circ \tau_{n_{2}} \circ \ldots$ be a characteristic Arnoux-Rauzy sequence containing $u$ as its $r$ th bispecial factor, where we order the bispecial factors of $\nu$ according to increasing length. Fix $a \in A_{k}$ and let $\omega$ be any characteristic Arnoux-Rauzy sequence whose $S$-adic expansion begins with $\tau_{n_{1}} \circ \tau_{n_{2}} \circ \ldots \tau_{n_{r}} \circ \tau_{a}$. Then in [27] it is proved that $\omega$ has the same first $r$ bispecial factors of $\nu$ (the $r$ th bispecial factor of a characteristic Arnoux-Rauzy sequence is completely determined by the first $r$ terms of its $S$-adic expansion), and that $a u$ is a right special factor of $\omega$ (the $r+1$ st term of the $S$-adic expansion of $\omega$ determines which of the $k$ factors $1 u, 2 u, \ldots, k u$ is right special in $\omega$ ).

Corollary 2. Let $b(n)$ denote the number of bispecial factors of $\mathcal{A R}$ of length $n$. Then

$$
r(n)=r(n-1)+(k-1) b(n-1) .
$$

Proof. In fact each bispecial factor of length $n-1$ is a suffix of $k$ right special factors of length $n$.

Combining Corollaries 1 and 2 we have: 
Corollary 3. Fix $k$ and let $\mathcal{A R}_{n}$ denote the set of all Arnoux-Rauzy words of length $n$ on the alphabet $A_{k}=\{1, \ldots, k\}$. Let $b(n)$ denote the number of bispecial words in $\mathcal{A R}$ of length $n$. Then

$$
\operatorname{Card}\left(\mathcal{A R}_{n}\right)=k+(n-1) k(k-1)+(k-1)^{2} \sum_{i=1}^{n-2}(n-i-1) b(i) .
$$

Proof. By Corollaries 1 and 2 we have

$$
\begin{aligned}
\operatorname{Card}\left(\mathcal{A R}_{n}\right) & =k+(k-1) \sum_{i=1}^{n-1} r(i) \\
& =k+(k-1) \sum_{i=1}^{n-1}\left(k+(k-1) \sum_{j=1}^{i-1} b(j)\right) \\
& =k+(n-1) k(k-1)+(k-1)^{2} \sum_{i=1}^{n-1} \sum_{j=1}^{i-1} b(j) \\
& =k+(n-1) k(k-1)+(k-1)^{2} \sum_{i=1}^{n-2}(n-i-1) b(i) .
\end{aligned}
$$

As a special case of Corollary 3 we recover the formula for the number of Sturmian words of length $n$.

Corollary 4. The number $\mathrm{St}_{n}$ of Sturmian words of length $n$ is

$$
1+\sum_{i=1}^{n}(n-i+1) \varphi(i)
$$

where $\varphi(i)$ is the Euler phi function.

Proof. Applying Corollary 3 to the case $k=2$ and the fact that $b(i)=$ $\varphi(i+2)$ (see [24] or Corollary 5 ahead) gives

$$
\begin{aligned}
\mathrm{St}_{n} & =2 n+\sum_{i=1}^{n-2}(n-i-1) b(i)=2 n+\sum_{j=3}^{n}(n-j+1) \varphi(j) \\
& =1+\sum_{j=1}^{n}(n-j+1) \varphi(j)
\end{aligned}
$$

where the last step follows from the equality $\varphi(1)=\varphi(2)=1$.

3. A generalization of the Euler phi function. In the Sturmian case we have $b(n)=\varphi(n+2)$. We now give a general arithmetic interpretation for the quantity $b(n)$ in terms of a multidimensional generalization of the Euclidean algorithm.

Fix $k$ and let

$$
E=\left\{\left(x_{1}, \ldots, x_{k}\right) \text { : each } x_{i} \text { is a nonnegative integer }\right\} .
$$


For $z=\left(x_{1}, \ldots, x_{k}\right) \in E$ set $|z|=\sum_{i=1}^{k} x_{i}$. Define a function $f: E \rightarrow E$ as follows: For $z=\left(x_{1}, \ldots, x_{k}\right) \in E$ fix the least $1 \leq j \leq k$ such that $x_{j} \leq x_{i}$ for all $1 \leq i \leq k$ and set

$$
f(z)=\left(x_{1}-x_{j}, \ldots, x_{j-1}-x_{j}, x_{j}, x_{j+1}-x_{j}, \ldots, x_{k}-x_{j}\right) .
$$

Clearly for each $z \in E$ there exists a (unique) vector $\widetilde{f}(z) \in E$ such that $f^{n}(z)=\widetilde{f}(z)$ for all $n$ sufficiently large. For $z \in E$ define the generalized greatest common divisor of $z$, denoted $\operatorname{ggcd}(z)$, by

$$
\operatorname{ggcd}(z)=|\widetilde{f}(z)| \text {. }
$$

For instance, $f(4,2,5)=(2,2,3)$ and $f(2,2,3)=(2,0,1)$ so that $\widetilde{f}(4,2,5)=$ $(2,0,1)$ and $\operatorname{ggcd}(4,2,5)=3$. For $k=2$ it follows immediately from the definition that $\operatorname{ggcd}(a, b)=\operatorname{gcd}(a, b)$.

To the best of our knowledge, this algorithm was first defined in [5] (in the special case $k=3$ ) in connection with a generalization of the Fine-Wilf theorem to three periods.

Set $P=\left\{z=\left(x_{1}, \ldots, x_{k}\right) \in E: \operatorname{ggcd}(z)=1\right\}$ and $P(n)=\{z \in P$ : $|z|=n\}$. Then we have

Theorem 1. Fix $k$ and let $\mathcal{A R}$ denote the set of all Arnoux-Rauzy words on the alphabet $A_{k}=\{1, \ldots, k\}$. Let $b(n)$ denote the number of bispecial words $u \in \mathcal{A R}$ of length $n$. Then $b(n)=\operatorname{Card} P((k-1) n+k)$.

Proof. Let $B(n)$ denote the set of bispecial words in $\mathcal{A R}$ of length $n$, so that $b(n)=$ Card $B(n)$. For each $n \geq 1$ we construct a bijection $\psi_{n}$ : $B(n) \rightarrow P((k-1) n+k)$ as follows: Let $u \in B(n)$; according to Lemma 2, the word $u$ is a bispecial factor of some Arnoux-Rauzy sequence $\omega \in \mathcal{A R}_{\infty}$. For each $1 \leq i \leq k$ let $v$ (possibly the empty word) denote the longest proper prefix of $u$ so that $i v$ is a right special factor of $\omega$. If such a $v$ exists, set $x_{i}=|| u|-| v||$. If no such $v$ exists, set $x_{i}=|u|+1$. It follows from the so-called "hat algorithm" given in Section III of [27] that for each $i$ the quantity $x_{i}$ is independent of the choice of $\omega$. Set $\psi_{n}(u)=\left(x_{1}, \ldots, x_{k}\right)$.

We now show that $\psi_{n}: B(n) \rightarrow P((k-1) n+k)$ and is a bijection for each $n$. Taking $n=1$ we have $B(1)=A_{k}=\{1, \ldots, k\}$. Fixing $i \in B(1)$ we see by definition of $\psi_{1}$ that $\psi_{1}(i)$ is the vector whose $i$ th coordinate is 1 and all other coordinates are 2 , so that $\left|\psi_{1}(i)\right|=(k-1) 2+1=(k-1) 1+k$ as required. Moreover $f\left(\psi_{1}(i)\right)=(1,1, \ldots, 1)$ and $f^{2}\left(\psi_{1}(i)\right)=(1,0,0, \ldots, 0)$ so that $\operatorname{ggcd}\left(\psi_{1}(i)\right)=1$. Clearly $\psi_{1}$ is injective. To see that $\psi_{1}$ is also surjective, let $z=\left(x_{1}, \ldots, x_{k}\right) \in P((k-1) 2+1)$. Hence $|z|=(k-1) 2+1$ and $\operatorname{ggcd}(z)=1$. These conditions clearly imply that each $x_{i}>0$. If all $x_{i} \geq 2$ we would have $|z| \geq 2 k$, a contradiction. Hence some $x_{i}=1$. We claim that all other coordinates of $z$ are 2. In fact, if $x_{s} \neq x_{t}$ for some choice of $s \neq i$ and $t \neq i$, then $\widetilde{f}(z)$ would have two nonzero coordinates, contradicting the fact that $\operatorname{ggcd}(z)=1$. Hence $z$ is a vector whose $i$ th coordinate is 1 and all 
other coordinates are equal to one another. As $|z|=(k-1) 2+1$, the other coordinates of $z$ must all be 2 , whence it follows that $z=\psi_{1}(i)$ as required.

Now let $n>1$ and suppose that $\psi_{m}: B(m) \rightarrow P((k-1) m+k)$ is a bijection for all $m<n$. Let $u$ be a bispecial word of length $n$. We begin by showing that $\psi_{n}(u)=\left(x_{1}, \ldots, x_{k}\right)$ as defined above is in $P((k-1) n+k)$. Fix an Arnoux-Rauzy sequence $\omega$ in which $u$ is a bispecial factor. Let $v$ denote the longest proper prefix of $u$ which is also bispecial in $\omega$ and fix $i \in A_{k}$ so that $i v$ is a right special factor of $\omega$. Hence $x_{i}<x_{j}$ for all $1 \leq j \leq k$. Then by definition of $\psi_{n}$ we have $x_{i}=|| u|-| v||$. Set $\psi_{|v|}(v)=\left(y_{1}, \ldots, y_{k}\right)$. Thus $y_{i}=x_{i}$, in fact $u=v v^{\prime}$ where $v^{\prime}$ is a suffix of $v$ of length $x_{i}$ (see the hat algorithm in Section III of [27]). Moreover, for $j \neq i$ we have $y_{j}=x_{j}-x_{i}$. Hence $\psi_{|v|}(v)=f\left(\psi_{n}(u)\right)=\left(x_{1}-x_{i}, \ldots, x_{i-1}-x_{i}, x_{i}, x_{i+1}-x_{i}, \ldots, x_{k}-x_{i}\right)$. By the inductive hypothesis we have $\operatorname{ggcd}\left(\psi_{|v|}(v)\right)=1\left(\right.$ and hence $\left.\operatorname{ggcd}\left(\psi_{n}(u)\right)=1\right)$ and $\left|\psi_{|v|}(v)\right|=(k-1)|v|+k=(k-1)\left(|u|-x_{i}\right)+k$, whence $\left|\psi_{n}(u)\right|=$ $\sum_{j=1}^{k} x_{j}=(k-1)|u|+k$ as required. Hence $\psi_{n}(B(n)) \subset P((k-1) n+k)$.

If for some $u^{\prime} \in B(n)$ with $u^{\prime} \neq u$ we had $\psi_{n}(u)=\psi_{n}\left(u^{\prime}\right)$ then $\psi_{|v|}$ (where $v$ is as above) would fail to be injective on $B(|v|)$. Hence $\psi_{n}$ is one-to-one. To see that $\psi_{n}$ is a surjection, let $z=\left(x_{1}, \ldots, x_{k}\right) \in P((k-1) n+k)$. Thus $|z|=(k-1) n+k$ and $\operatorname{ggcd}(z)=1$. As in the case $n=1$ these conditions imply that each $x_{j}>0$. Fix $i$ such that $x_{i} \leq x_{j}$ for all $1 \leq j \leq k$. We claim that $x_{i}<x_{j}$ for all $j \neq i$. In fact, if for some $j \neq i$ we had $x_{j}=x_{i}$, then $f(z)$ would have a coordinate equal to zero. Since $\operatorname{ggcd}(z)=1$ this would imply that $z=(1,1, \ldots, 1)$, contradicting $|z|=(k-1) n+k$. Consider $f(z)=\left(x_{1}-x_{i}, \ldots, x_{i-1}-x_{i}, x_{i}, x_{i+1}-x_{i}, \ldots, x_{k}-x_{i}\right)$. Then $\operatorname{ggcd}(f(z))=1$ (since $\operatorname{ggcd}(z)=1)$ and hence $f(z) \in P\left((k-1)\left(n-x_{i}\right)+k\right)$. By the inductive hypothesis, since $\psi_{n-x_{i}}: B\left(n-x_{i}\right) \rightarrow P\left((k-1)\left(n-x_{i}\right)+k\right)$ is onto, we have $f(z)=\psi_{n-x_{i}}(v)$ for some bispecial word $v \in \mathcal{A R}$ of length $n-x_{i}$. Let $\omega$ be any Arnoux-Rauzy sequence containing $i v$ as a right special factor, and let $u$ be the shortest bispecial factor of $\omega$ beginning with $v i$. Then it follows from the hat algorithm that $u=v v^{\prime}$ where $v^{\prime}$ is a suffix of $v$ of length $x_{i}$ (see Section III of [27]). Hence $|u|=n$ and $\psi_{n}(u)=\left(x_{1}, \ldots, x_{k}\right)=z$ as required.

As a special case of Theorem 1 we have:

Corollary 5. The number of bispecial Sturmian words of length $n$ is $\varphi(n+2)$, where $\varphi$ denotes the Euler phi function.

Proof. Applying Theorem 1 to the case $k=2$ gives $b(n)=\operatorname{Card} P(n+2)$. But

$$
\begin{aligned}
P(n+2) & =\{(a, b) \in E \mid a+b=n+2 \text { and } \operatorname{ggcd}(a, b)=1\} \\
& =\{(a, n+2-a) \in E \mid \operatorname{gcd}(a, n+2-a)=1\} \\
& =\{(a, n+2-a) \in E \mid \operatorname{gcd}(a, n+2)=1\}
\end{aligned}
$$


hence $\operatorname{Card} P(n+2)=\operatorname{Card}\{a \mid 1 \leq a<n+2$ and $\operatorname{gcd}(a, n+2)=1\}=$ $\varphi(n+2)$.

From Theorem 1 and Corollary 3 we deduce:

Corollary 6. Fix $k$ and let $\mathcal{A R}_{n}$ denote the set of all Arnoux-Rauzy words of length $n$ on the alphabet $A_{k}=\{1, \ldots, k\}$. Then

$\operatorname{Card}\left(\mathcal{A R}_{n}\right)=k+(n-1) k(k-1)+(k-1)^{2} \sum_{i=1}^{n-2}(n-i-1) \operatorname{Card} P((k-1) i+k)$.

REMARK 1. In [24], following a suggestion of G. Rauzy, Mignosi establishes a connection between the number of Sturmian words, Farey numbers, and the Riemann hypothesis. Another such connection between the Riemann hypothesis, the Euler phi function and the formula in Corollary 4 was given by Bender, Patashnik, and Rumsey [3] using a result of Codèca [10]. It would be interesting to find similar connections involving the multidimensional generalization of the Euler phi function described in this paper for $k>2$, two-dimensional Farey numbers in the sense of [2, 29, 30], and deep results and conjectures in analytical number theory. Also, Rychlik points out a possible connection between our multidimensional generalization of the Euler phi function and Gröbner bases [28].

\section{References}

[1] P. Arnoux and S. Ito, Pisot substitutions and Rauzy fractals, Bull. Belgian Math. Soc. Simon Stevin 8 (2001), 181-207.

[2] P. Arnoux et G. Rauzy, Représentation géométrique de suites de complexité $2 n+1$, Bull. Soc. Math. France 119 (1991), 199-215.

[3] E. A. Bender, O. Patashnik and H. Rumsey, Pizza slicing, phi's, and the Riemann hypothesis, Amer. Math. Monthly 101 (1994), 307-317.

[4] J. Cassaigne, S. Ferenczi and L. Q. Zamboni, Imbalances in Arnoux-Rauzy sequences, Ann. Inst. Fourier (Grenoble) 50 (2000), 1265-1276.

[5] M. G. Castelli, F. Mignosi and A. Restivo, Fine and Wilf's theorem for three periods and a generalization of Sturmian words, Theoret. Comput. Sci. 218 (1999), 83-94.

[6] R. V. Chacon, Weakly mixing transformations which are not strongly mixing, Proc. Amer. Math. Soc. 22 (1969), 559-562.

[7] N. Chekhova, Propriétés combinatoires des suites d'Arnoux-Rauzy et réponse à une question d'Hedlund et Morse, preprint, 1999.

[8] -, Algorithme d'approximation et propriétés ergodiques des suites d'Arnoux-Rauzy, preprint, 1999.

[9] N. Chekhova, P. Hubert et A. Messaoudi, Propriétés combinatoires, ergodiques et arithmétiques de la substitution de Tribonacci, J. Théor. Nombres Bordeaux, to appear.

[10] P. Codèca, A note on Euler's $\varphi$ function, Ark. Mat. 19 (1981), 261-263.

[11] E. Coven and G. A. Hedlund, Sequences with minimal block growth, Math. Systems Theory 7 (1973), 138-153. 
[12] X. Droubay, J. Justin and G. Pirillo, Epi-Sturmian words and some constructions of de Luca and Rauzy, Theoret. Comput. Sci. 255 (2001), 539-553.

[13] S. Dulucq et D. Gouyou-Beauchamps, Sur les facteurs des suites de Sturm, ibid. 71 (1990), 381-400.

[14] S. Ferenczi, Bounded remainder sets, Acta Arith. 61 (1992), 319-326.

[15] -, Les transformations de Chacon: combinatoire, structure géométrique, lien avec les systèmes de complexité $2 n+1$, Bull. Soc. Math. France 123 (1995), 271-292.

[16] S. Ferenczi, C. Holton and L. Q. Zamboni, The structure of 3-interval exchange transformations I: An arithmetic study, Ann. Inst. Fourier (Grenoble) 51 (2001), 861-901.

[17] - - - - , The structure of 3-interval exchange transformations II: Combinatorial properties, preprint, 2001.

[18] - - - - , The structure of 3-interval exchange transformations III: Ergodic and spectral properties, preprint, 2001.

[19] S. Ferenczi and C. Mauduit, Transcendence of numbers with a low complexity expansion, J. Number Theory 67 (1997), 146-161.

[20] T. Kamae and L. Q. Zamboni, Maximal pattern complexity of discrete dynamical systems, Ergodic Theory Dynamical Systems, to appear.

[21] H. Kesten, On a conjecture of Erdös and Szüsz related to uniform distribution mod 1, Acta Arith. 12 (1966), 193-212.

[22] M. Lothaire, Algebraic Combinatorics on Words, Cambridge Univ. Press, to appear. Available at http://www-igm.univ-mlv.fr/berstel.

[23] A. de Luca and F. Mignosi, Some combinatorial properties of Sturmian words, Theoret. Comput. Sci. 136 (1994), 361-385.

[24] F. Mignosi, On the number of factors of sturmian words, Theoret. Comput. Sci. 82 (1991), 71-84.

[25] M. Morse and G. A. Hedlund, Symbolic dynamics II: Sturmian sequences, Amer. J. Math. 62 (1940), 1-42.

[26] G. Rauzy, Nombres algébriques et substitutions, Bull. Soc. Math. France 110 (1982), $147-178$.

[27] R. N. Risley and L. Q. Zamboni, A generalization of Sturmian sequences: Combinatorial structure and transcendence, Acta Arith. 95 (2000), 167-184.

[28] M. Rychlik, personal communication (2001).

[29] N. Wozny and L. Q. Zamboni, Frequencies of factors in Arnoux-Rauzy sequences, Acta Arith. 96 (2001), 261-278.

[30] L. Q. Zamboni, Une généralisation du théorème de Lagrange sur le développement en fraction continue, C. R. Acad. Sci. Paris Sér. I 327 (1998), 527-530.

Dipartimento di Matematica e Applicazioni

Università degli Studi di Palermo

Via Archirafi 34

90123 Palermo, Italia

Department of Mathematics University of North Texas

E-mail: mignosi@dipmat.math.unipa.it 